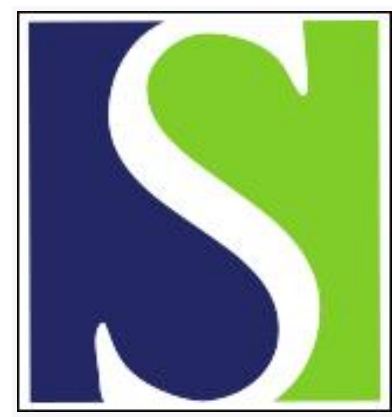

Scand J Work Environ Health 2011;37(6):533-538

https://doi.org/10.5271/sjweh.3164

Published online: 15 Apr 2011, Issue date: Nov 2011

Maternal shift work during pregnancy and biomarkers of reproductive function among the male offspring - a pilot follow-up study

by Ramlau-Hansen CH, Håkonsen LB, Christensen M, Bonde JP, Olsen J, Thulstrup AM

Affiliation: The Danish Ramazzini Center, Department of Occupational Medicine, Aarhus University Hospital, Noerrebrogade 44, Building 2C, 8000 Aarhus C, Denmark. ceciraml@rm.dk

Refers to the following texts of the Journal: 1999;25(2):85-99 2008;34(1):5-22 1997;23(4):257-265

Key terms: biomarker; maternal shift work; occupational health; offspring; pilot study; pregnancy; prenatal exposure; reproductive function; reproductive hormones; semen quality; shift work; sperm count

This article in PubMed: www.ncbi.nlm.nih.gov/pubmed/21499670 


\title{
Maternal shift work during pregnancy and biomarkers of reproductive function among the male offspring - a pilot follow-up study
}

\author{
Cecilia Høst Ramlau-Hansen, PhD, ${ }^{1,2}$ Linn Berger Håkonsen, ${ }^{1}$ Maria Christensen, ${ }^{1}$ Jens Peter Bonde, \\ PhD, ${ }^{3}$ Jorn Olsen, PhD, ${ }^{4}$ Ane Marie Thulstrup, $P h D^{1}$
}

\begin{abstract}
Ramlau-Hansen CH, Håkonsen LB, Christensen M, Bonde JP, Olsen J, Thulstrup AM. Maternal shift work during pregnancy and biomarkers of reproductive function among the male offspring - a pilot follow-up study. Scand $J$ Work Environ Health. 2011;37(6):533-538. doi:10.5271/sjweh.3164
\end{abstract}

Objectives The aim of this study was to examine the associations between maternal shift work during pregnancy and measures of semen quantity and quality and level of reproductive hormones among young, adult men.

Methods From a Danish pregnancy cohort established in 1984-1987, 347 sons aged 18-21 years were selected in 2005-2006 and semen and blood samples were taken. At around the $36^{\text {th }}$ week of gestation, the mothers completed a questionnaire that included a question on shift work during pregnancy. Information on shift work was available for mothers of 278 sons.

Results Of the 278 sons, 42 (15\%) had had fetal exposure to maternal shift work. Men exposed to maternal shift work had 30\% [95\% confidence interval (95\% CI) -58-16] lower adjusted percentage normal morphology sperm, 18\% (95\% CI -30-100) higher adjusted sperm concentration, and 11\% (95\% CI -0.6-25) higher adjusted levels of testosterone than men not exposed. Adjusted semen volume, total sperm count, percentage motile sperm and serum levels of estradiol, follicle stimulation hormone (FSH), luteinizing hormone (LH), inhibin B, and sex hormone-binding globulin (SHBG) were not associated with maternal shift work during pregnancy.

Conclusions The results of this pilot study indicate no strong associations between maternal shift work during pregnancy and the quantity and quality of semen or level of reproductive hormones among sons.

Key terms occupational health; prenatal exposure; reproductive hormones; semen quality; sperm count.

Shift work and working nights or evenings have been associated with an increased risk of reproductive dysfunction (1), adverse pregnancy outcomes [pre-term birth, spontaneous abortion (2), and low birth weight (3)] as well as gastrointestinal complaints (4), coronary heart disease (5-9), and breast cancer $(7,10,11)$. Shift work may disrupt the circadian rhythm, which controls body temperature and hormonal signalling $(6,12-14)$.

Exposure to light at night is associated with lower levels of melatonin, resulting in increased release of estrogen from the ovaries (15). A reduced amount of sleep, that can be caused by shift work, may also be associated with higher estradiol levels (16). Shift work has also been found to be associated with lower levels of testosterone (17). Increased prenatal exposure to estrogens or imbalance between estrogen and androgen in fetal life is suggested to be an important etiologic factor in male reproductive disorders like testicular cancer, cryptorchidism, hypospadias and low sperm count $(18,19)$. However, to our knowledge, no studies have examined if maternal shift work during pregnancy may cause hormonal disruption and adverse development of male fetal gonads.

The objective of this study was to investigate the associations between maternal shift work during pregnancy, semen quantity and quality, and the level of reproductive hormones among sons. We conducted a population-based cohort study of young adult men.

1 The Danish Ramazzini Center, Department of Occupational Medicine, Aarhus University Hospital, Aarhus, Denmark.

2 Department of Epidemiology, Institute of Public Health, University of Aarhus, Aarhus, Denmark.

3 Department of Occupational and Environmental Medicine, Copenhagen University Hospital Bispebjerg, Copenhagen, Denmark.

4 UCLA School of Public Health, Department of Epidemiology, Los Angeles, CA, USA.

Correspondence to: Cecilia Høst Ramlau-Hansen, The Danish Ramazzini Center, Department of Occupational Medicine, Aarhus University Hospital, Noerrebrogade 44, Building 2C, 8000 Aarhus C, Denmark. [E-mail: ceciraml@rm.dk] 


\section{Methods}

\section{Study population}

The study participants were the sons of mothers who were recruited to the "Healthy Habits for Two" cohort during their pregnancy from 1984-1987 (20). The study took place in two Danish municipalities (Aalborg and Odense), and 11980 women with singleton pregnancies (more than $80 \%$ of all invited) participated. They provided information on health-related characteristics and lifestyle factors, including information on employment, during pregnancy around the $36^{\text {th }}$ week of gestation. Sons who were alive and living in Denmark by December 2004 were identified in the Danish Civil Registration System $(\mathrm{N}=5109)$.

Since the data collection was designed for a study on prenatal smoking exposure and adult semen quality (21), the participants were selected according to levels of maternal smoking during pregnancy. Letters of invitation were sent consecutively, and the oldest and those living close to either Aalborg (north of Jutland) or Odense (Funen) had priority, starting at the city centres and up to approximately $30 \mathrm{~km}$ from the centres. Having a limited number of men heavily exposed to prenatal tobacco smoke resulted in use of an expanded geographic area for this group. Additionally, men whose mothers had reported information on health-related issues from childhood to adolescence by means of a selfadministered questionnaire when the sons were 16-19 years of age were given priority. A total of 716 men were invited to take part in the study, and 347 (48.5\%) gave consent and participated. Of 100 men who declined participation by mail or telephone, 82 provided some information on their health. Information on maternal shift work during pregnancy was available for 278 sons, who constitute the study population.

The selected participants were 18-21 years of age and received an economic compensation (DKr 500) for responding to the questionnaires and providing a semen and blood sample. Men with severe handicaps or syndromes, such as spastic paraplegia or Down's syndrome, as well as men with metabolic diseases or psychiatric disorders, were not included in the study. The Region Committees on Biomedical Research Ethics in Denmark approved the study (registration number 20040174), and participation was conditional on written informed consent.

\section{Exposure data}

Information on employment, including shift work, during pregnancy was obtained in the "Healthy Habits for Two" study by self-administered questionnaire handed out around the $36^{\text {th }}$ week of gestation, filled out at home and returned in sealed envelopes to the university's research department within a couple of weeks.

The mothers were asked whether they had been working during pregnancy. If so, they were asked "Have you been doing shift work during your pregnancy?" The response categories were "Yes", "No", "No answer".

\section{Outcome data}

Sampling of semen and blood samples took place between February 2005-January 2006. The subjects were instructed to abstain from ejaculation in the 48 hours prior to semen sampling. The samples were collected by masturbation into a plastic container at home and then brought to a mobile laboratory positioned at the participants' nearest university hospital, where a trained medical laboratory technician performed the initial semen analysis blinded toward any prenatal conditions. The participants also completed questionnaires on reproductive, medical, and lifestyle factors (eg, smoking), time and date of the preceding ejaculation and spillage during collection of the semen sample.

Semen volume was estimated by its weight $(1 \mathrm{~g}=1 \mathrm{ml})$. Sperm motility and sperm concentration were assessed as described in the World Health Organization's Laboratory Manual for the Examination of Human Semen and Sperm-Cervical Mucus Interaction (22). Examination of $82 \%$ of the samples was initiated within one hour of ejaculation, and examination of $99.7 \%$ of the samples was initiated within two hours. Sperm morphology was determined using the Tygerberg strict criteria (23). The laboratory took part in the European Society for Human Reproduction and Embryology's external quality control (EQC) programme, and all control tests were in agreement with results obtained by expert examiners within the EQC programme.

Blood samples were taken between 07:25-19:15 hours (median time: 13:05 hours), and after centrifugation, serum was stored at $-80^{\circ} \mathrm{C}$ for a maximum of $16^{1 / 2}$ months until analyzed blinded toward any prenatal conditions. Serum samples for testosterone, estradiol, follicle stimulation hormone (FSH) and luteinizing hormone (LH) were analyzed by Avida Centaur (Bayer Healthcare, Leverkusen, Germany). The sex hormone-binding globulin (SHBG) concentrations were determined using IMMULITE (DPC, Koege, Denmark), and inhibin B was measured by a commercially available enzymelinked immunosorbent assay (Oxford Bio-Innovation Ltd, Oxford, UK) according to the manufacturer's instructions.

\section{Statistical analysis}

Crude median, $25^{\text {th }}$ and $75^{\text {th }}$ percentiles were calculated for all outcome variables. For each of the outcomes, we performed multiple linear regression analysis to 
test for differences between exposure groups, taking a priori selected potential confounders identified in the literature into account. Data on the outcome variables were transformed by use of the natural logarithm before multiple linear regressions were performed, and the estimates were back-transformed to the original scale and presented as relative differences with $95 \%$ confidence intervals $(95 \% \mathrm{CI})$ of the adjusted medians. To obtain a more symmetric distribution of residuals, we also cubicroot transformed all the outcome variables (with the exception of percentages of motile sperm, which were logit transformed, and inhibin B, which were not transformed). The risk estimates were, however, essentially the same as in analyses based on the natural logarithm. Results were adjusted for (i) season (summer/winter), (ii) history of diseases of the reproductive organs among sons (cryptorchidism, hypospadias, varicocele, hydrocele, orchitis, and chlamydia combined into one variable, present or not present), (iii) the young men's smoking habits (yes/no), and (iv) maternal smoking during pregnancy (yes/no). The semen outcome variables were additionally adjusted for abstinence time $(\leq 48$ hours, 49 hours -5 days, $>5$ days) and spillage during collection of the sample (yes, no). Data on participants who reported spillage during masturbation $(\mathrm{N}=73)$ were excluded from all statistical analysis on semen volume and total sperm count. The results on motility were additionally adjusted for time from ejaculation to analysis (continuous, in minutes). Blood sample outcome were additionally adjusted for time of day of blood sampling (06:00-08:59, 09:00-12:00, or later than 12:00 hours).

\section{Results}

The characteristics of the 278 participants according to maternal shift work during pregnancy are presented in table 1 . Sons of mothers who did not work shifts during pregnancy $[\mathrm{N}=236(85 \%)]$ had a lower body mass index than sons of mothers with shift work $[\mathrm{N}=42(15 \%)]$, but otherwise the two groups were quite similar. Of the shift working pregnant women, 23 (55\%) were employed in healthcare.

Men exposed to maternal shift work had 30\% (95\% CI -58-16) lower adjusted percentage normal morphology sperm and 18\% (95\% CI -30-100) higher adjusted sperm concentration than unexposed men (table 2). None of these differences were statistically significant. Semen volume, total sperm count and percentage motile sperm were not associated with maternal shift work during pregnancy.

Exposed men had 11\% (95\% CI -0.6-25) higher adjusted testosterone (table 2), a borderline statistically significant finding. Exposed men had a crude median $\left[25^{\text {th }}-75^{\text {th }}\right.$ percentiles $\left.(\mathrm{p} 25-75)\right] \mathrm{LH}$ of $5.0 \mathrm{iu} / 1$ (p25-75: 3.7-6.2) in comparison with $4.2 \mathrm{iu} / 1$ (p25-75: 3.3-5.3) among unexposed men, corresponding to a difference of $19 \%(\mathrm{P}=0.04)$. However, this difference disappeared after transformation and adjustment. Likewise, there were no associations between maternal shift work during pregnancy and adjusted levels of estradiol, FSH, inhibin $\mathrm{B}$, or SHBG.

\section{Discussion}

We did not observe any strong or significant associations between maternal shift work during pregnancy and semen quantity and quality or level of reproductive hormones among male offspring. For most outcome variables, adjusted differences between exposure groups were $\leq 10 \%$. Findings for sperm morphology, sperm concentration, and serum testosterone are somewhat contradictory, indicating higher means of sperm concentration and testosterone and lower mean of normal morphology sperm of men exposed to maternal shift work in fetal life in comparison with men not exposed. Low sperm counts are expected to be associated with low levels of gonadotrophins if the cause is the pituitary or central nervous system (CNS) level and with compensatory high levels of gonadotrophins if the cause is at the testicular level. Levels of testosterone and sperm counts are expected to correlate positively. Our findings do not support the hypothesis that maternal shift work during pregnancy has strong negative effect at neither fetal pituitary, CNS, nor testicular level. However, confidence intervals are wide, and the results must be interpreted with caution.

The participation rate was $48.5 \%$, which is a fairly high rate for a semen quality study (the normal rate in such a study is $25-50 \%$ ) (24), but not high enough to eliminate the risk of selection bias. If our results would be explained by selection bias towards the null, sons of shift working mothers with good semen quality or sons of not shift working mothers with poor semen quality would have been over sampled. However, the population of sons was young, most had no reproductive experience, and they were not aware of the hypothesis evaluated, facts which reduce, but do not eliminate, the risk of selection bias. We compared participants and non-participants who completed a small questionnaire on health $(\mathrm{N}=82)$ and found no difference in the proportion of men with diseases of the reproductive organs (including cryptorchidism and hypospadias).

Information on shift work was obtained through a questionnaire completed late in the pregnancy, and therefore risk of differential misclassification of the reporting of shift work is limited. Non-differential misclassification is nevertheless possible and may have 
Table 1. Characteristics of 278 Danish young men according to maternal shift work during pregnancy [SD=standard deviation]

\begin{tabular}{|c|c|c|c|c|c|c|c|c|}
\hline & \multicolumn{8}{|c|}{ Maternal shift work during pregnancy } \\
\hline & \multicolumn{4}{|c|}{ Yes $(\mathrm{N}=42)$} & \multicolumn{4}{|c|}{ No $(\mathrm{N}=236)$} \\
\hline & Mean & SD & $\mathrm{N}$ & $\%$ & Mean & SD & $\mathrm{N}$ & $\%$ \\
\hline \multicolumn{9}{|l|}{ Person-related characteristics of sons } \\
\hline Body mass index $\left(\mathrm{kg} / \mathrm{m}^{2}\right)$ & 24.4 & 3.8 & & & 23.2 & 3.5 & & \\
\hline Birth weight $(\mathrm{g})$ & 3403 & 520 & & & 3518 & 522 & & \\
\hline History of diseases in reproductive organs ${ }^{\text {a }}$ & & & 5 & 12 & & & 43 & 18 \\
\hline Recent fever & & & 4 & 10 & & & 37 & 16 \\
\hline Cigarette smoking, daily & & & 20 & 48 & & & 86 & 36 \\
\hline Alcohol consumption, weekly & & & 18 & 43 & & & 115 & 59 \\
\hline \multicolumn{9}{|l|}{ Person-related characteristics of mothers } \\
\hline Mother smoking during pregnancy & & & 29 & 69 & & & 166 & 70 \\
\hline Mother drinking alcohol during pregnancy & & & 36 & 86 & & & 198 & 84 \\
\hline \multicolumn{9}{|l|}{ Semen- and blood-related characteristics } \\
\hline \multicolumn{9}{|l|}{ Season } \\
\hline October-March & & & 15 & 36 & & & 94 & 40 \\
\hline April-September & & & 27 & 64 & & & 142 & 60 \\
\hline \multicolumn{9}{|l|}{ Duration of abstinence } \\
\hline$\leq 48$ hours & & & 17 & 40 & & & 72 & 31 \\
\hline 49 hours -5 days & & & 23 & 55 & & & 149 & 63 \\
\hline$>5$ days & & & 2 & 5 & & & 15 & 6 \\
\hline Minutes from ejaculation to start of analysis & 52 & 19 & & & 52 & 20 & & \\
\hline Spillage at semen sampling & & & 12 & 29 & & & 61 & 26 \\
\hline Blood collected between $06.00-12.00$ & & & 17 & 40 & & & 87 & 37 \\
\hline
\end{tabular}

a History of diseases in the reproductive organs include cryptorchidism, hypospadias, varicocele, hydrocele, orchiditis, and chlamydial infection combined into one variable (present or not present).

Table 2. Semen and hormonal characteristics for 278 Danish young men according to maternal shift work during pregnancy. [p25-p $75=25^{\text {th }}-75^{\text {th }}$ percentiles; $95 \% \mathrm{Cl}=95 \%$ confidence interval.]

\begin{tabular}{|c|c|c|c|c|c|c|c|c|}
\hline \multirow[t]{2}{*}{ Parameter } & \multicolumn{2}{|c|}{$\begin{array}{l}\text { No maternal shift work } \\
\qquad(\mathrm{N}=236)\end{array}$} & \multicolumn{2}{|c|}{$\begin{array}{l}\text { Maternal shift work } \\
\qquad(\mathrm{N}=42)\end{array}$} & \multicolumn{2}{|c|}{$\begin{array}{l}\text { Maternal shift work relative } \\
\text { to no maternal shift work } \\
\text { (reference) }\end{array}$} & \multicolumn{2}{|c|}{ P-value a } \\
\hline & Median & p25-p75 & Median & p25-p75 & $\begin{array}{l}\text { Adjusted }{ }^{b} \\
\text { percentage } \\
\text { difference }\end{array}$ & $95 \% \mathrm{Cl}$ & $\begin{array}{l}\text { Crude } \\
\text { median }\end{array}$ & $\begin{array}{l}\text { Adjusted } \\
\text { geometric } \\
\text { medians }\end{array}$ \\
\hline Sperm concentration (millions/ml) & 40 & $20-90$ & 41 & $22-68$ & 18 & $-30-100$ & 0.84 & 0.54 \\
\hline Semen volume $(\mathrm{ml})^{c}$ & 3.0 & $2.2-3.9$ & 3.2 & $2.3-4.1$ & -2 & $-19-19$ & 0.78 & 0.85 \\
\hline Sperm total count (millions) ${ }^{c}$ & 121 & $48-291$ & 109 & $54-284$ & 3 & $-43-86$ & 0.94 & 0.92 \\
\hline Percent normal morphology sperm & 6.0 & $3.0-9.0$ & 4.5 & $2.0-7.0$ & -30 & $-58-16$ & 0.09 & 0.17 \\
\hline Percent motile sperm & 71 & $61-77$ & 67 & $64-76$ & 8 & $-15-37$ & 0.70 & 0.53 \\
\hline Testosterone (nmol/l) & 17 & $13-20$ & 18 & $14-24$ & 11 & $-0.6-25$ & 0.05 & 0.06 \\
\hline Estradiol (nmol/l) & 0.10 & $0.07-0.12$ & 0.11 & $0.07-0.15$ & 10 & $-4-27$ & 0.16 & 0.16 \\
\hline $\begin{array}{l}\text { Sex hormone-binding globulin (SHBG) } \\
\text { (nmol/l) }\end{array}$ & 26 & $20-33$ & 28 & $21-38$ & 6 & $-7-20$ & 0.39 & 0.40 \\
\hline $\begin{array}{l}\text { Follicle stimulation hormone (FSH) } \\
\text { (iu/l) }\end{array}$ & 2.9 & $2.1-4.2$ & 3.1 & $2.1-4.7$ & 7 & $-17-37$ & 0.49 & 0.61 \\
\hline Luteinizing hormone (LH) (iu/l) & 4.2 & $3.3-5.3$ & 5.0 & $3.7-6.2$ & -8 & $-25-13$ & 0.04 & 0.44 \\
\hline Inhibin B (ng/ml) & 152 & $115-182$ & 150 & 103-182 & -7 & $-19-7$ & 0.49 & 0.31 \\
\hline
\end{tabular}

a Differences between groups were tested by Wilcoxon rank sum test (crude medians) and multiple linear regression analysis (adjusted medians).

b Percentage differences between medians of the two groups stratified on maternal shift work during pregnancy, no shift work constituting the reference group. Differences are adjusted for season (summer/winter), history of diseases of the reproductive organs (present, not present), smoking (yes/no) and maternal smoking during pregnancy (yes/no). The semen outcome variables were additionally adjusted for abstinence time ( $\leq 48$ hours, 49 hours-5 days, $>5$ days) and spillage during collection of the sample (yes/no). The results for motility were also adjusted for minutes from ejaculation to analysis (continuous). Blood sample outcome were additionally adjusted for time of day of blood sampling (06:00-08:59, 09:00-12:00, or later than 12:00 hours).

${ }^{c}$ Participants reporting spillage during collection were excluded $(\mathrm{N}=73)$. 
led to bias toward the null, since some of the unexposed could have been shift working in early pregnancy, the time period that is most critical in fetal gonadal development. Only few will start shift working in late pregnancy, and women reporting shift work late in pregnancy probably also did shift work early in the pregnancy. The semen and blood samples were examined blinded toward any prenatal exposure, so differential misclassification of the outcome variables is unlikely.

Different mechanisms on how shift work can cause disease have been discussed. In cancer research, a "light-at night" hypothesis has been discussed. Light at night suppresses melatonin production, which may lead to accumulation of oxygen radicals and possible DNA damage (25). It has also been suspected that light increases the production of oestrogen through the production of FSH and LH, and shift work may associated with lower levels of testosterone (17).

The estrogen hypothesis leads to the prediction that prenatal conditions associated with high estrogen levels would lead to increased risk of, for example, low sperm counts (18). A review of the published epidemiologic studies on indicators of prenatal serum levels of maternal estrogens with male reproductive disorders published in 2005 indicates a lack of strong evidence for the hypothesis, except for testicular cancer (26), and it may be the imbalance between estrogen and androgen - rather than increased estrogen exposure - that causes decreased sperm counts (19).

In conclusion, the results of this pilot study indicate no strong association between maternal shift work during pregnancy and the quantity and quality of semen or level of reproductive hormones among sons. Future studies should be larger and consider timing and type of shift work.

\section{References}

1. Labyak S, Lava S, Turek F, Zee P. Effects of shiftwork on sleep and menstrual function in nurses. Health Care Women Int. 2002;23:703-14. doi:10.1080/07399330290107449.

2. Whelan EA, Lawson CC, Grajewski B, Hibert EN, Spiegelman D, Rich-Edwards JW. Work schedule during pregnancy and spontaneous abortion. Epidemiology. 2007;18:350-5. doi:10.1097/01.ede.0000259988.77314.a4.

3. Schlunssen V, Viskum S, Omland O, Bonde JP. Medfører skiftarbejde øget forekomst af spontan abort, præterm fødsel eller lav fødselsvægt? [Does shift work cause spontaneous abortion, preterm birth or low birth weight?]. Ugeskr Laeger. 2007;169:893-900.

4. Burch JB, Tom J, Zhai Y, Criswell L, Leo E, Ogoussan K. Shiftwork impacts and adaptation among health care workers. Occup Med (Lond). 2009;59:159-66. doi:10.1093/occmed/ kqp015.
5. Boggild H, Knutsson A. Shift work, risk factors and cardiovascular disease. Scand J Work Environ Health. 1999;25:85-99.

6. Costa G. The problem: shiftwork. Chronobiol Int. 1997;14:89 98. doi:10.3109/07420529709001147.

7. Knutsson A. Health disorders of shift workers. Occup Med (Lond). 2003;53:103-8.doi:10.1093/occmed/kqg048.

8. Olsen O, Kristensen TS. Impact of work environment on cardiovascular diseases in Denmark. J Epidemiol Community Health. 1991;45:4-9. doi:10.1136/jech.45.1.4.

9. Tenkanen L, Sjoblom T, Kalimo R, Alikoski T, Harma M. Shift work, occupation and coronary heart disease over 6 years of follow-up in the Helsinki Heart Study. Scand J Work Environ Health. 1997;23:257-65.

10. Davis S, Mirick DK, Stevens RG. Night shift work, light at night, and risk of breast cancer. J Natl Cancer Inst. 2001;93:1557-62. doi:10.1093/jnci/93.20.1557.

11. Kolstad HA. Nightshift work and risk of breast cancer and other cancers--a critical review of the epidemiologic evidence. Scand J Work Environ Health. 2008;34:5-22.

12. Andersen E. Health effects of shiftwork--a focus on health care providers. AAOHN J. 2005;53:239-40.

13. Biggi N, Consonni D, Galluzzo V, Sogliani M, Costa G. Metabolic syndrome in permanent night workers. Chronobiol Int. 2008;25:443-54. doi:10.1080/07420520802114193.

14. Costa G, Sartori S, Akerstedt T. Influence of flexibility and variability of working hours on health and well-being. Chronobiol Int. 2006;23:1125-37.n doi:10.1080/07420520601087491.

15. Mirick DK, Davis S. Melatonin as a biomarker of circadian dysregulation. Cancer Epidemiol Biomarkers Prev. 2008;17:3306-13. doi:10.1158/1055-9965.EPI-08-0605.

16. Mahoney MM. Shift work, jet lag, and female reproduction. Int J Endocrinol. 2010;2010:813764.

17. Axelsson J, Akerstedt T, Kecklund G, Lindqvist A, Attefors R. Hormonal changes in satisfied and dissatisfied shift workers across a shift cycle. J Appl Physiol. 2003;95:2099-105.

18. Sharpe RM, Skakkebaek NE. Are oestrogens involved in falling sperm counts and disorders of the male reproductive tract? Lancet. 1993;341:1392-5. doi:10.1016/01406736(93)90953-E.

19. Sharpe RM. The 'oestrogen hypothesis'- where do we stand now? Int J Androl. 2003;26:2-15.doi:10.1046/j.13652605.2003.00367.x.

20. Olsen J, Frische G, Poulsen AO, Kirchheiner H. Changing smoking, drinking, and eating behaviour among pregnant women in Denmark. Evaluation of a health campaign in a local region. Scand J Soc Med. 1989;17:277-80.

21. Ramlau-Hansen CH, Thulstrup AM, Storgaard L, Toft G, Olsen J, Bonde JP. Is prenatal exposure to tobacco smoking a cause of poor semen quality? A follow-up study. Am J Epidemiol. 2007;165:1372-9.doi:10.1093/aje/kwm032.

22. World Health Organization. WHO Laboratory Manual for the Examination of Human Semen and Sperm-Cervical Mucus Interaction. 4th ed. Cambridge: Cambridge University Press; 1999. 
23. Kruger TF, Acosta AA, Simmons KF, Swanson RJ, Matta JF, Oehninger S. Predictive value of abnormal sperm morphology in in vitro fertilization. Fertil Steril. 1988;49:112-7.

24. Larsen SB, Abell A, Bonde JP. Selection bias in occupational sperm studies. Am J Epidemiol. 1998;147:681-5.

25. Kantermann T, Roenneberg T. Is light-at-night a health risk factor or a health risk predictor? Chronobiol Int. 2009;26:1069-74.
26. Storgaard L, Bonde JP, Olsen J. Male reproductive disorders in humans and prenatal indicators of estrogen exposure. A review of published epidemiological studies. Reprod Toxicol. 2006;21:4-15.doi:10.1016/j.reprotox.2005.05.006.

Received for publication: 30 August 2010 